\title{
CONCENTRATED AND RARIFIED SETS OF POINTS.
}

BY

\author{
A. S. BESICOVITCH
}

Trinity College, CAmbridge.

\section{CHAPTER I.}

\section{Concentrated Sets.}

I have arrived at the definition of concentrated sets from the following two similar problems:

Problem I. What are the linear sets whose measure with respect to any con tinuous monotone function $\varphi(l)$ is zero?

Problem II. What are the linear sets on which the variation of any continuous monotone function is zero?

Concentrated sets are defined as follows:

A non-enumerable set of points $E$ is said to be concentrated in the neighbourhood of an enumerable set $H$ if any open set containing the set $H$ contains also the set $E$ with the exception of at most an enumerable set of points.

$\S 1$. Measure with respect to a function. Let $\varphi(l)$ be a positive increasing function satisfying defined for $l>0$ and such that $\varphi(+o)=0$ and let $E$ be a linear set of points. Denote by $I=\Sigma l_{i}$ any sequence of intervals containing the whole of the set $E$ ( $l_{i}$ denoting both an interval and its length). Denote by $m_{\varphi}^{\lambda} E$ the lower bound of the sum

$$
\sum \varphi\left(l_{i}\right)
$$

37-33617. Acta mathematica. 62. Imprimé le 12 février 1934. 
for all possible sets $I$, for which the length of the largest interval does not exceed $\lambda$. The limit of $m_{\varphi}^{i} E$, as $\lambda$ tends to zero, we shall call the exterior $\varphi$-measure of $E$, or the exterior measure of $E$ with respect to $\varphi$.

Obviously the $\varphi$-measure of an enumerable set and the variation of a continuous monotone function with respect to an enumerable set are both zero. Now we know that a $B$-measurable set $E$ either is enumerable or else contains a perfect subset $E^{\prime}$. In the first case the variation of any monotone continuous function on $E$ is zero. In the second case $E^{\prime}$ may be transformed into an interval by means of a monotone continuous function $f(x)$. The length of such an interval is obviously the variation of $f(x)$ with respect to $E^{\prime}$, and hence the variation with respect to $E$ is positive. Hence

The only B-measurable sets whose rariation with respect to any continuous function is zero are enumerable sets. ${ }^{1}$

We shall shew that

In the general case there are non-enumerable sets whose measure is zero with respect to any function $\varphi$,

but the proof will be based on the assumption that the power of the continuum is $\mathbf{w}_{1}$.

§. We shall first prove some preliminary theorems.

Theorem 1. A necessary and sufficient condition for a linear set $E$ to be of measure zero with respect to any function $\varphi(l)$ is that given any decreasing sequence of positive numbers

$$
l_{i}, \quad i=\mathrm{I}, 2, \ldots,
$$

however rapidly tending to zero, there always exists a sequence $I$ of intervals $\left(x_{i}^{\prime}, x_{i}^{\prime \prime}\right), \quad x_{i}^{\prime \prime}-x_{i}^{\prime} \leqq l_{i}$, containing the whole of the set $E$.

The condition is necessary. For let $E$ be a set of measure zero with respect to any function and $l_{i} \quad(i=\mathrm{I}, 2, \ldots)$ assume that there exists any decreasing

${ }^{1}$ L. C. Young. Note on the theory of measure. Proceedings of the Cambridge Philosophical Society. Vol: XXVI. Part 1.

L. C. Young considers variation of a function on a given set as measure of this set. The definition we are using is not so general and it is not obvious that if variation of some functions on a given set is positive then also measure of the set with respect to some function is positive. We shall consider in $\$ 3 B$-measurable sets of measure zero with respect to any function. 
sequence of positive numbers. Define an increasing function $\varphi(l)$ satisfying the conditions

$$
\varphi\left(l_{i}\right)=\frac{\mathrm{I}}{i} \quad \text { for } i=\mathrm{I}, 2, \ldots
$$

Obviously $\varphi(+0)=0$, and consequently $m_{\varphi} E$ is defined and by hypothesis is equal to zero. Therefore there exists a set $I$ of intervals $\left(x_{i}^{\prime}, x_{i}^{\prime \prime}\right), x_{i}^{\prime \prime}-x_{i}^{\prime}=l_{i}^{\prime}$, decreasing in length, containing the whole of the set $E$ and such that

$$
\sum \varphi\left(l_{i}^{\prime}\right)<\mathrm{I} .
$$

Then for any $n$

i. $e$.

$$
\begin{gathered}
\text { I }>\sum_{i=1}^{n} \varphi\left(l_{i}^{\prime}\right) \geqq n \varphi\left(l_{n}^{\prime}\right), \\
\varphi\left(l_{n}^{\prime}\right)<\frac{\mathrm{I}}{n}
\end{gathered}
$$

$$
l_{n}^{\prime}<l_{n}
$$

and the condition is therefore satisfied.

The condition is also sufficient. For suppose a set $\boldsymbol{E}$ satisfies this condition: then given a function $\varphi(l)$ satisfying the usual conditions and two positive constants $\lambda, \varepsilon$ we can always find a sequence of positive numbers $l_{i}<\lambda$ such that

$$
\sum \varphi\left(l_{i}\right)<\varepsilon
$$

and then a sequence $I$ of intervals $\left(x_{i}^{\prime}, x_{i}^{\prime \prime}\right), x_{i}^{\prime \prime}-x_{i}^{\prime}=l_{i}$, containing $E$, which shews that measure of $E$ with respect to $\varphi(l)$ is zero.

$\S 3$. We can now solve the problem mentioned in the footnote to $\S 1$.

Theorem 2. The only B-measurable sets of measure zero with respect to any function are enumerable sets. $F$ r any perfect set $\boldsymbol{P}$ there exists a function $\varphi(l)$ with respect to which the measure of the set is positive.

On account of 2 the theorem will be proved if we prove that given any perfect set $P$ there exists a sequence of positive numbers $l_{n}, n=1,2, \ldots$ such that no set of intervals of lengths $l_{1}, l_{2}, \ldots$ can cover the whole of the set $P$. Obviously the set $P$ cannot be covered with one interval of arbitrarily small 
length. Let $\lambda_{1}$ be the lower bound of the length of an interval which covers the whole of $P$ (evidently $\lambda_{1}$ is the distance between the extreme points of $P$ ). Take for $l_{1}$ an arbitrary positive number less than $\lambda_{1}$.

It is also easy to shew that the set $E$ cannot be covered with one interval of length $l_{1}$ and another interval of arbitrarily small length. Otherwise let two intervals

$$
\left(x_{n}, x_{n}+l_{1}\right), \quad\left(y_{n}, y_{n}+l_{2, n}\right)
$$

where $l_{2, n} \rightarrow 0$, as $n \rightarrow \infty$, cover the whole of $P$ for any $n$. Let $(x, y)$ be a limit point of $\left(x_{n}, y_{n}\right)$. Then for any $\varepsilon$ the two intervals

$$
\left(x-\varepsilon, x+l_{1}+\varepsilon\right), \quad(y-\varepsilon, y+\varepsilon)
$$

cover $P$. Consequently the set $P$ is included in the set consisting of the interval $\left(x, x+l_{1}\right)$ and of the point $y$. Being a perfect set, $P$ has no isolated point and thus is contained in the interval $\left(x, x+l_{1}\right)$, which is impossible.

Let $\lambda_{z}$ be the lower bound of the length of an interval which together with another interval of length $l_{1}$ covers the whole of the set $P$. Denoting by $l_{2}$ a positive number less than $\lambda_{z}$ we have that no pair of intervals of lengths $l_{1}, l_{2}$ can cover the whole of the set $P$. Similarly we can find $l_{3}>0$ such that no three intervals of lengths $l_{1}, l_{2}, l_{3}$ can cover the whole of $P$, and so on. Thus we arrive at a sequence of positive numbers

$$
l_{1}, l_{2}, \ldots
$$

such that no finite set of intervals whose lengths are represented by different numbers of the sequence, can cover the set $P$. Then by the Heine-Borel theorem no infinite set of intervals of lengths

$$
l_{1}, l_{2}, \ldots
$$

can cover the set $P$, which proves the theorem.

$\S 4$. Theorem 3. If the measure of a set $E$ is zero with respect to any function $p(l)$, then the variation of any monotone continuous function $f(x)$ on $E$ is zero.

Denote by $O\{f(x), l\}$ the upper bound of the oscillation of $f(x)$ on any interval of length $l$. Obviously

$$
O\{f(x), l\} \rightarrow 0, \quad \text { as } l \rightarrow 0 .
$$

Consequently, given $\varepsilon>0$ we can always find a decreasing sequence of positive numbers $l_{i}, \quad i=1,2, \ldots$ such that 


$$
\sum_{i=1}^{\infty} 0\left\{f(x), l_{i}\right\}<\varepsilon
$$

On the other hand, by Theorem I the set $E$ can be included in a set of intervals $\left(x_{i}^{\prime}, x_{i}^{\prime \prime}\right), x_{i}^{\prime \prime}-x_{i}^{\prime}<l_{i}$. Then denoting by $V_{f} E$ the variation of $f(x)$ on the set $E$ we have

$$
\begin{aligned}
V_{f} E & \leqq \sum\left\{f\left(x_{i}^{\prime \prime}\right)-f\left(x_{i}^{\prime}\right)\right\} \\
& \leqq \sum O\left\{f(x), l_{i}\right\} \\
& <\varepsilon
\end{aligned}
$$

which proves the theorem.

After these preliminary theorems have been proved we can pass to the main problem of this note, i.e., to the proof of the existence of non-enumerable sets of measure zero with respect to any function $\varphi(l)$. It can be easily shewn that concentrated sets, if they exist, are of measure zero with respect to any function $\varphi(l)$. For let $E$ be a set concentrated in the neighbourhood of a set $\left\{x_{n}\right\} \quad(n=\mathrm{I}, 2, \ldots)$ and $\left\{l_{n}\right\}$ an arbitrary sequence of positive numbers. By the definition the set of intervals $\left(x_{n}-\frac{\mathrm{I}}{2} l_{2 n}, x_{n}+\frac{\mathrm{I}}{2} l_{2 n}\right)$ contains all points of $E$ except at most an enumerable set of them, which can be included in intervals of length $l_{1}, l_{3}, \ldots$ Consequently by Theorem $\mathrm{I}$ the set $E$ is of measure zero with respect to any function.

Thus our problem reduces to the proof of the existence of concentrated sets.

$\S 5$. This proof will be based on the existence of a transfinite sequence $\left\{\varphi_{i}\right\}$ of positive decreasing functions which will be called a fundamental sequence.

We say that a function $f(n)$ is ultimately greater than (greater than or equal to) another function $g(n)$, and we write

$$
\begin{gathered}
f(n) \succ g(n) \\
(f(n) \succcurlyeq g(n)),
\end{gathered}
$$

if there exists a number $n_{0}$ such that 
for $n>n_{0}$.

$$
\begin{gathered}
f(n)>g(n) \\
(f(n) \geqq g(n))
\end{gathered}
$$

Definition. A sequence $\left\{\varphi_{i}(n)\right\}$, where $i$ takes all values less than $\omega_{1}, \omega_{1}$ being the first transfinite number of power $\mathbf{\Sigma}_{1}$, is called a fundamental sequence if any positive function $f(n)$ ultimately greater than all $\varphi_{i}(n)$ except at most an enumerable set of them.

The existence of such a sequence can be established in the following way. We first order the set of all positive decreasing functions into a sequence $f_{i}(n)$ $i$ taking all values less than $\omega_{1}$.

We then define

and so on.

$$
\begin{aligned}
& \varphi_{1}(n)=f_{1}(n) \\
& \varphi_{2}(n)=\min \left\{\varphi_{1}(n), f_{2}(n)\right\} \\
& \varphi_{3}(n)=\min \left\{\varphi_{\geq}(n), f_{3}(n)\right\}
\end{aligned}
$$

For any transfinite number $i+1$ of the first class we put

$$
\varphi_{i+1}(n)=\min \left\{\varphi_{i}(n), f_{i+1}(n)\right\} .
$$

For any transfinite number $i$ of the second class we first define a sequence $k_{l} \quad\left(l<\omega_{0}, \omega_{0}\right.$ being the first transfinite number of power $\left.\boldsymbol{\aleph}_{0}\right)$ of increasing transfinite numbers such that

$$
\lim k_{l}=i \text {. }
$$

Then we define $\varphi_{i}(l)$ for any $l$ by the equation

$$
\varphi_{i}(l)=\min \left\{f_{i}(l), \varphi_{k_{1}}(l), \ldots, \varphi_{k_{l}}(l)\right\} .
$$

In this way the sequence

$$
\varphi_{i}(l)
$$

is defined, where $i$ takes all values less than $\omega_{1}$. The sequence possesses the following properties:

(i) For any $i<\omega_{1}$

$$
f_{i}(n) \geqq \varphi_{i}(n)
$$

(ii) Any $\varphi_{i}(n)$ is a decreasing function. 
(iii) If $i<k$, then

$$
\varphi_{i}(n) \succeq \varphi_{k}(n)
$$

The properties (i), (ii) are obvious. In order to prove (iii) we observe that

$$
\varphi_{1}(n) \succeq \varphi_{2}(n) \succeq \varphi_{3}(n) \ldots
$$

Suppose that these inequalities have been proved for all indices less than $i$. Then if $i$ is a transfinite number of the first class, we have

$$
\varphi_{i-1}(n) \succeq \varphi_{i}(n)
$$

by the definition of $\varphi_{i}(n)$. If $i$ is of the second class, then

$$
i=\lim k_{l}
$$

and again from the definition of $\varphi_{i}(n)$ we conclude that

$$
\varphi_{k_{l}}(n) \succeq \varphi_{i}(n)
$$

for all $l$, and consequently

for any $j<i$.

$$
\varphi_{j}(n) \succcurlyeq \varphi_{i}(n)
$$

(iv) To any transfinite number $i<\omega_{1}$ corresponds an $i^{\prime}>i$ and less than $\omega_{1}$, such that

$$
\varphi_{i}(n) \succ \varphi_{i^{\prime}}(n)
$$

For, all decreasing functions having been enumerated we have

$$
\frac{\mathrm{I}}{2} \varphi_{i}(n)=f_{i^{\prime}}(n)
$$

where $i^{\prime}$ must be greater than $i$ since $\varphi_{i}(n) \succ f_{i^{\prime}}(n)$. As $f_{i^{\prime}}(n) \succeq \varphi_{i^{\prime}}(n)$ we have

$$
\varphi_{i}(n) \succ \varphi_{i^{\prime}}(n)
$$

Let now $g(n)$ be any positive function.

Define $f(n)$ by the condition

$$
f(n)=\min g(m) \quad \text { for } \mathbf{I} \leqq m \leqq n .
$$

$f(n)$ is a decreasing function and consequently

and thus

$$
f(n)=f_{i}(n), \quad i<\omega_{1}
$$

$$
f(n) \succeq \varphi_{i}(n)
$$

and as there exists $i^{\prime}>i$ such that $\varphi_{i}(n) \succ \varphi_{i^{\prime}}(n)$ we have that 


$$
f(n)>\varphi_{j}(n) \quad \text { for all } j \geqq i^{\prime}
$$

$i$.e., for all $j$ except an enumerable set of values. Thus the sequence

is fundamental.

$$
\left\{\varphi_{i}(n)\right\}
$$

§ 6. Lemma. Let $\left\{x_{n}\right\},\left(n<\omega_{0}\right)$, be a set of points everywhere dense, $\left\{y_{n}\right\}$ any enumerable set of points and $f(n)$ any positice function. There always exists a point a different from all $y_{n}$ and such that the inequality

$$
\left|\alpha-x_{n}\right|<f(n)
$$

is satisfied for infinitely many values of $n$.

Take an arbitrary positive integer $n_{1}$ and the interval $\left(x_{n_{1}}-\lambda_{1}, x_{n_{1}}+\lambda_{1}\right)$, where $\lambda_{1}<f\left(n_{1}\right)$ and is so small that the interval does not contain any of the numbers $y_{1}, y_{2}, \ldots, y_{n_{1}}$ different from $x_{n_{1}}$. Take now an integer $n_{2}>n_{1}$ and a positive number $\lambda_{2}<f\left(n_{2}\right)$ such that the interval $\left(x_{n_{3}}-\lambda_{2}, x_{n_{2}}+\lambda_{2}\right)$ is contained in the interval $\left(x_{n_{1}}-\lambda_{1}, x_{n_{1}}+\lambda_{1}\right)$ and does not contain any of the numbers $y_{1}, y_{2}, \ldots, y_{n_{2}}$ different from $x_{n_{2}}$, and so on.

In this way we arrive at a sequence of intervals

$$
\left(x_{n_{i}}-\lambda_{i}, x_{n_{i}}+\lambda_{i}\right) \quad i=\mathrm{I}, 2, \ldots<\omega_{0}
$$

each of them being interior to the preceding one. Any point $\alpha$ interior to all these intervals (if $\lambda_{i} \rightarrow 0$ there is only one such point) is different from all $y_{n}$ and satisfies the conditions

$$
\begin{aligned}
\left|\alpha-x_{n_{i}}\right| & <\lambda_{i} \\
& <f\left(n_{i}\right) \quad \text { for } i=\mathrm{I}, 2, \ldots
\end{aligned}
$$

which proves the lemma.

$\S 7$. We shall now construct a concentrated set of points of power $\aleph_{1}$.

Let $\varphi_{i}(n),\left(i<\omega_{1}\right)$, be a fundamental sequence of functions and $\left\{x_{n}\right\}$ an everywhere dense set of points. Take a point $\alpha_{1}$ different from all points $x_{n}$ and such that the inequality

$$
\left|\alpha_{1}-x_{n}\right|<\varphi_{1}(n)
$$

is satisfied for infinitely many values of $n$. Then choose a point $\alpha_{2}$ different from all $x_{n}$ and from $\alpha_{1}$ and such that the inequality

$$
\left|\alpha_{2}-x_{n}\right|<\varphi_{2}(n)
$$


is satisfied for infinitely many values of $n$. When $\alpha_{k}$ has been defined for all $k<i$ we define $\alpha_{i}$ to be different from all $x_{n}$ and from all $\alpha_{k}, k<i$, and to satisfy the inequality $\left|\alpha_{i}-x_{n}\right|<\varphi_{i}(n)$ for infinitely many values of $n$. Thus we arrive at a set of points $\left\{\alpha_{i}\right\},\left(i<\omega_{1}\right)$.

Let now $\left\{l_{n}\right\}$ be an arbitrary sequence of positive numbers. Take the set of intervals $\left(x_{n}-l_{n}, x_{n}+l_{n}\right), n=\mathrm{I}, 2, \ldots$ and write $f(n)=l_{n}$. Let $i_{0}$ be the least transfinite number such that

$$
f(n) \succ \varphi_{i_{0}}(n) .
$$

Take now any $i>i_{0}$. We know that

$$
\left|\alpha_{i}-x_{n}\right|<\varphi_{i}(n)
$$

for infinitely many values of $n$ and thus

$$
\left|\alpha_{i}-x_{n}\right|<f(n)
$$

for infinitely many values of $n$. Thus among all the intervals $\left(x_{n}-l_{n}, x_{n}+l_{n}\right)$ there are infinitely many ones containing $\alpha_{i}$ for any $i>i_{0}$. The set of points $\alpha_{1}, \ldots \alpha_{i_{0}}$ being enumerable we conclude that the set of points $\alpha_{i},\left(i<\omega_{1}\right)$, is concentrated in the neighbourhood of the set $\left\{x_{n}\right\}$ and thus our problem is solved.

\section{CHAPTER II}

\section{Rarified Sets.}

I have arrived at the definition of rarified sets from the following:

Problem. Has every plane set of infinite linear measure a subset of finite measure?

This seems obvious and yet it is not the case.

We shall construct a linearly measurable plane set of infinite measure, every subset of which is either of infinite measure or of measure zero.

The solution will be given by rarified sets. They are defined as follows:

A non-enumerable plane set is said to be rarified if any subset of this set, of plane measure zero, consists of at most an enumerable set of points.

The construction of most rarified sets will be based on the assumption that the power of the continuum is $\boldsymbol{s}_{1}$.

Denote by $E$ any set of plane measure zero which can be represented by the product

38-33617. Acta mathematica. 62. Imprimé le 18 avril 1934. 


$$
E=\prod_{1}^{\infty} A_{i}
$$

where each $A_{i}$ is an open set. As any open set is defined by an enumerable set of points (for instance by the points with rational coordinates belonging to the set), then so is any set $E$.

Now the aggregate of all enumerable sets of points on a plane is known to have the power of the continuum. Hence the aggregate of all the sets $E$ has power of continum.

Let now two transfinite sequences $E_{i}, P_{i}, \quad i$ running through all values of power less than $\aleph_{1}$, represent all the sets $E$ and all perfect sets of positive plane measure, belonging to the square $(\mathrm{o} \leqq x \leqq 1,0 \leqq y \leqq 1)$.

Denote by $M_{1}$ an arbitrary point of the set

$$
P_{1}-E_{1} P_{1}
$$

by $M_{2}$ an arbitrary point of the set

$$
P_{2}-\left(E_{1}+E_{2}\right) P_{2}
$$

different from $\boldsymbol{M}_{1}$, by $\boldsymbol{M}_{3}$ an arbitrary point of

$$
P_{3}-\left(E_{1}+E_{2}+E_{3}\right) P_{3}
$$

different from $\boldsymbol{M}_{1}$ and $\boldsymbol{M}_{2}$, and so on. After we have defined $\boldsymbol{M}_{i}$ for all $i<i_{0}$, $M_{i_{0}}$ will be defined as an arbitrary point of

$$
P_{i_{0}}-\left(E_{1}+E_{\mathrm{q}}+\cdots+E_{i_{0}}\right) P_{i_{0}}
$$

different from all the points $M_{i}$ for $i<i_{0}$. Such a point exists for any $i_{0}<\omega_{1}$. For, the set

$$
E_{1}+E_{2}+\cdots+E_{i_{0}}
$$

is of plane measure zero, since it is the sum of an enumerable set of sets, each of plane measure zero. On the other hand $P_{i_{0}}$ is of positive plane measure and consequently so is the set

$$
P_{i_{0}}-\left(E_{1}+E_{2}+\cdots+E_{i_{1}}\right) P_{i_{0}}
$$

and thus it contains more than an enumerable set of points. Hence it must contain points different from an enumerable set of points $\boldsymbol{M}_{i},\left(i<i_{0}\right)$.

Denote by $G$ the set of all the points $M_{i}$. 
(i) The exterior plane measure of $G$ is $\mathrm{I}$.

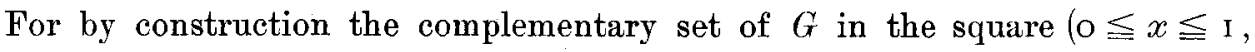
$\mathrm{O} \leqq y \leqq \mathrm{I}$ ) does not contain any perfect subset of positive plane measure.

Corollary. The exterior linear measure of $G$ is infinite.

(ii) The intersection of $G$ with any set $E_{i_{0}}$ is an enumerable set.

For the points of $G$ contained in $E_{i_{0}}$ are all among the points $M_{i}$ $\left(i=\mathrm{I}, 2, \ldots<i_{0}\right)$.

(iii) Any subset of $G$ of plane measure zero is an enumerable set.

For, any such set is contained in a set $E_{i}$.

Corollary 1. G is a rarified set.

Corollary 2. The set $G$ is linearly measurable.

For let $H$ be any set of finite positive exterior linear measure $\mu^{*} H, a$ fortiori of plane measure zero.

The set $G \cdot H$ being enumerable we have

$$
\begin{gathered}
\mu^{*}(H-G \cdot H)=\mu^{*} H \\
\mu^{*} G \cdot H=\mathrm{o}
\end{gathered}
$$

and thus

$$
\mu^{*} H=\mu^{*}(H-G \cdot H)+\mu^{*} G \cdot H
$$

which proves the corollary.

Theorem. The set $G$ has no subset of finite positive exterior linear measure.

For let $H$ be a subset of $G$ of finite exterior linear measure. Then by (iii) $G: H$ is an enumerable set and so is $H$, since $G \cdot H=H$.

Thus the set $G$ gives a solution of the problem of this chapter.

Note some other properties of the set $G$.

(iv) The set $G$ being itself measurable has no non-measurable subset.

$(\nabla)$ The set $G$ is not measurable with respect to plane measure.

For if it were measurable its measure would be equal to its exterior measure, $i . e$, to I. Then by the Fubini theorem its intersection with allmost all lines of the square, parallel to the sides of the square, will be sets of linear measure I, which is impossible by (iii).

Thus $G$ gives an example of a set which is measurable with respect to linear. measure and non-measurable with respect to plane measure. 
The converse phenomenon is, of course, very trivial since any set lying on a straight line is of plane measure zero but need not be linearly measurable.

Remark. We can remove the above anomalous possibilities by introducing a new definition of measurable sets.

Definition. $A$ set $H$ is said to be linearly measurable if it can be represented by the difference of the product of a finite or enumerably infinite set of open sets and of a set of linear measure zero. 